\title{
Fuzzy Logic Evaluation of Personality Profile and Alexithymia with Emotional Suppression in Patients with Central Serous Retinopathy
}

\author{
Elham Foroozandeh ${ }^{1}$, Pouya Derakhshan-Barjoei ${ }^{2^{*}}$, Mojdeh Bahadorzadeh ${ }^{3}$ \\ $I^{*}$ Department of Psychology, Naein Branch, Islamic Azad University, Naein, Iran \\ ${ }^{2}$ Department of Electrical and Computer Engineering, Naein Branch, Islamic Azad University, Naein, Iran \\ ${ }^{3}$ Department of General Surgery, Qom University of Medical Sciences, Qom, Iran
}

*Corresponding Author: Pouya Derakhshan-Barjoei, Department of Electrical and Computer Engineering, Naein Branch, Islamic Azad University, Naein, Iran

\begin{abstract}
Central serous retinopathy, also known as central Chorioretinopathy, is a condition that usually causes visual impairment and often occurs in one eye. The origin of this disease as mentioned by Conrad and et. al.[1], is uncertain, but more common in men in the age group of 20 to 50 years, and sometimes in other age groups. In the last decade, consideration has been given to psychological factors such as personality, emotional characteristics of the disease. The purpose of this study is to determine the relationship between personality profile and alexithymia with emotional suppression in patients with central serous retinopathy. This evaluation is obtained by fuzzy logic algorithm by obtaining appropriate membership functions and fuzzy rules. On the one hand, as a result of Jimenez et. al.[2], psychological factors, especially problems with emotional adjustment, along with the long-term of sympathetic stimulation by stress and tension can cause to increase the risk of central serous retinopathy. Also psychological problems is one of lamentable result of central serous retinopathy after blindness apruptly
\end{abstract}

Keywords: Fuzzy Logic, Alexithymia, Emotional Suppression, Central Serous Retinopath

\section{INTRODUCTION}

Central serous retinopathy, also known as central chorioretinopathy, is an eye disease that usually causes temporary visual impairment and is often seen in one eye. When the disease is active, it is characterized by fluid leakage under the retina with a tendency to accumulate under the central yellow spot (macula). This leads to a dim view (Metamorphopsia). The presence of a dark or gray spot in the center of the field of view is common during retina detachment. Reducing visual acuity may persist after absorption of fluid [3]. Reviewing psychological disturbances and poor quality of life in patients with chorioretinopathy has been done in [4]. The aim of this study was to compare the mental health and quality of life of patients with chorioretinopathy. In this research, SCL-90 questionnaire was used to assess mental health. The result of this study was that patients with chorioretinopathy reported a low level of quality of life. It also showed that patients with chorioretinopathy had a poor quality of life.

In [5] investigation of the physiological effects of emotional suppression has been figured out. The results of this study showed that emotional suppression was associated with increased physiological activity due to increased physiological activity and reduction of muscle tone improvement. In [6], the results of study showed that diabetes-related stress has a mediator role between depression and the severity of type 1 and type 2 diabetes, while those who were not depressed had a significantly lower severity of type 1 and type 2 diabetes.For this purpose, two groups of healthy subjects and diabetics were selected. The results of the measurements have been reported in this way that peoples with diabetes significantly excited in their emotions and feelings. Investigated the lifestyle, personality and health characteristics of patients with psychiatric disorders in [7] has been done. The results showed that inappropriate lifestyle affects their mental health, and in this group, depression and anxiety had the highest prevalence, respectively. Also, in relation to the relationship between personality characteristics and mental health, the results of this study showed that patients with low mental health 
disorders had high neuroticism, low extraversion, low agreeableness, and low level of conscientious. Also in [8] the research was on exploration of the personality profile of individuals with alexithymia. The results of this study showed that individuals with alexithymia and non- alexithymia have distinct personality profiles. Individuals without alexithymia are extrinsic and optimistic and have high selfesteem and an internal control center, as opposed to those with alexithymia; they tend to have levels of neuroticism.

The role of alexithymia in depression and anxiety in students has been investigated in [9].

A significant relationship was determined between alexithymia and HbA1c, depression, and anxiety in [10].

On the other hand, according to [11], Alexithymia is the lack of expression of excitement due to a failure in the ability to process and regulate excitement, or to deliberately inhibit emotional expression, and therefore they consider alexithymia as a structure that is related to emotional control. In addition, the history of the study suggests that central serous retinopathy has a psychological component. On the other hand, the role of personality traits, alchemy, and emotional suppression has also been confirmed in various physical conditions. Therefore, the present study attempts to answer the question of whether there is a relationship between personality dimensions and alexithymia with anxiety disorder in patients with central serous retinopathy.

Despite critiques and controversies, by the late 80's fuzzy sets have achieved a degree of legitimacy in psychology [12]. One of these recent developments is our current modeling of fuzzy set theory and applications in psychology.

\section{RESEARCH METHOD AND EVALUATION}

The research method is descriptive and correlational. Regarding the aim of this study, the correlation method was used to examine the relationship between personality dimensions and Alexithymia with emotional suppression in patients with central serous retinopathy, and the possibility of controlling and manipulating variables.

The statistical population included all patients with central serous retinopathy (CSR) who visited the ophthalmology clinic in 2016.In this research, available sampling method was used. It was chosen from among the ophthalmic clinics. After describing the goals and the research process for the center's ophthalmologist, patients with central serous retinopathy referred to the hospital and the conditions for entry into the research, such as diagnosis by the eye specialist, the satisfaction of the patients in the research, primary school certification and no history of severe psychiatric disorders were evaluated. The emotional control questionnaire has four subscales of emotional suppression, aggression control, rumination, or mental review and benign control in 65 subjects. In fact, each subscale has 14 substances. The emotional control questionnaire score is based on the correct and incorrect answer, which is the correct option for score 1 and belongs to the wrong choice of zero score. The score for each person in the scale from zero to 14 and in the total scale will range from zero to 56. A higher score indicates more emotional control. The NEO-FFI Personality Inventory was created in 1999 by Goldberg [13], and according to the Costa and MacKey model. This questionnaire was developed to assess personality traits in five factors:neuroticism, extraversion, openness, and agreeableness and conscientious. The questionnaire consists of 60 items, and in front of each item, a five-point range is perfectly accurate from the 5 th to the totally incorrect score of 1 .

The Toronto Alexithymia Scale is a 20-question test and has three subtests [14]. The first subcategory consists of 7 materials that assess the subject's ability to identify emotions and distinguish between feelings and physical sensations. The second sub-scale measures 5 substances, which is the ability of a person to express feelings and whether he can express his feelings in the form of words. The third sub-scale has 8 substances and examines the degree of introspection and deepening of the individual in his inner feelings and others. The range of response to each item is 5 degrees and from the totally opposite to the totally agree, is the method of scoring in Linkert scale. The answer is quite opposite to the score of 1 , and the answer is exactly the same as the score of 5.

The descriptive statistics of the sample are shown in the graph below. 


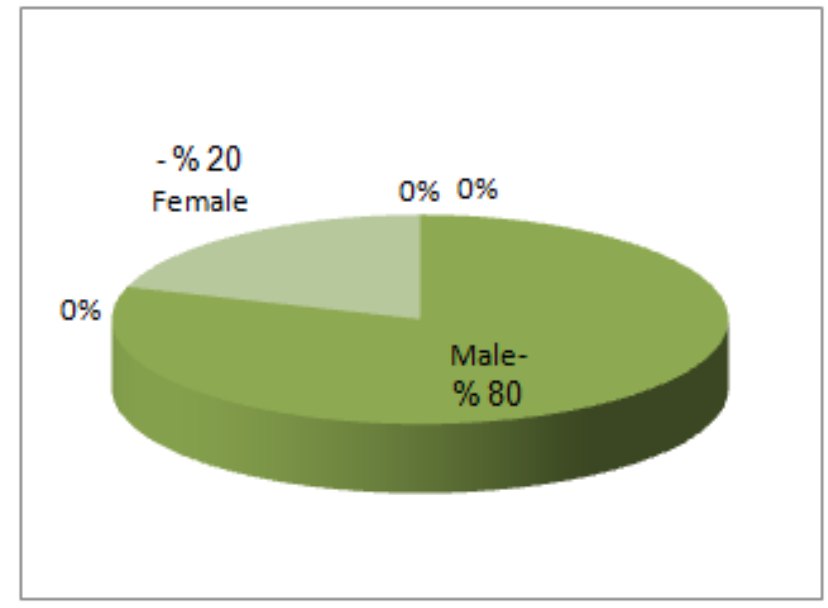

Fig1. Participants under study, 10 people are $20 \%$ female and $80 \%$ are male, so most people are male.

\section{FUZZY MODEL AND INFERENCE ENGINE}

It is used in psychological tests because it uses words inaccurately, such as very high - very low increasing or decreasing - reasonable - or somewhat reduced. All psychological tests are designed in this way. In other words, We can say that the answers of the questionnaires cannot be yes or no..The fuzzy logic first appeared in the scene of the New Testament for the first time in setting up the theory of fuzzy sets by Professor Lotfizadeh in 1965[15]. The fuzzy term means inaccurate, vague, or floating. The application of this section in software science can simply be defined so that fuzzy logic transcends the logic of zero values and one of the classical software and opens a new door for the world of software and computers, since the floating and infinite space between zero and one numbers also It uses and challenges its logic and arguments. It is already used in economics, psychology, linguistics, theology, and conflict resolution. One way to explain fuzzy logic is to compare it with Aristotelian logic. Aristotle considers the universe in terms of absolute logic, in black and white, while the fuzzy logic of Professor Lotfizadeh considers the universe in a variety of black and white shades. Scientifically, the real world is not discrete, but connected. In this math world, nothing is absolute. Everything varies to a certain extent on the scale 0 and 1 . As a result, there is between zero and one, tens and hundreds of other shades.New theories usually make fundamental changes in their areas. Professor Lotfizadeh's fuzzy logic is a brilliant example of this discussion [15-18].For each attribute specified by the patient, a membership function is assigned.Also, the evaluation results were made in the specified criteria for each specification. The fuzzy inference engine can also be selected from the category according to the best and most appropriate according to the characteristics. Below is an overview of a fuzzy system.

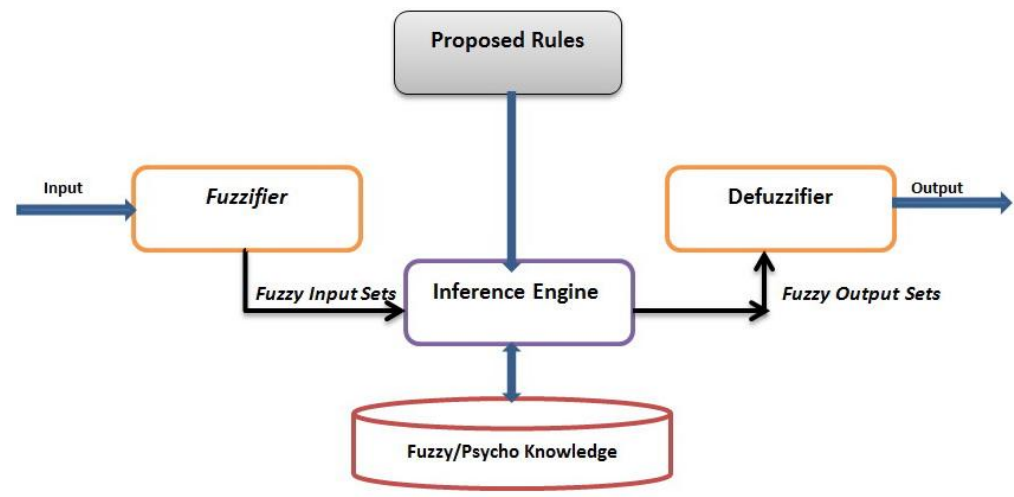

Fig2. Block diagram of general fuzzy system

In this model, we considered a fuzzy logic model for each test. In these models, by assigning the appropriate membership functions that we did in our previous research; we set up the fuzzy rules and rules table based on the input parameters and the scoring for the given solutions based on the rules of the law $[18,19]$. Considering the proper matching and obtaining optimal fuzzy membership functions, the responses and compliance with the patterns obtained and reviewed by the relevant specialist learning algorithm and the model were accepted. By placing the fuzzy output on the appropriate 
regions on the membership functions, there were acceptable ranges. According to the above description, the parameter set containing of the premise parameters determines a fuzzy classification system. Thus, different premise parameter sets determine different fuzzy classification systems resulting indifferent performances.Regarding the evaluation and determination of the relations in the form of fuzzy argument, an algorithm and an automated model were used to diagnose and determine the relation between the input and output of tests, which is the determination of the correlation between the parameters in the determined range.

In our proposed method we applied, PRODUCT method for inferencing, SUM method for composition and CENTROID method is used for defuzzification. A particular type of fuzzy inference system known as a Mamdani type is used. Fuzzy Inference Systems shows how the whole process works from beginning to end of evaluation process.

The function name is trimf which is used for triangular shaped member function. The degree of support is used to shape the output fuzzy set. Personality profile and alexithymia parameters mapped in the membership functions in the confined ranges.

\section{RESUlts}

The results of the correlation coefficient test showed that in the whole sample, there were significant relationships between, the extraversion and the dimensions of Alexithymia, and the difficulty in describing and identifying emotions with emotional suppression. In addition, the results showed that in the female sample, conscientiousness has a significant relationship with emotional suppression. Alexithymia and none of its dimensions had a significant relationship with emotional suppression. Also, in the men's sample from five dimensions of personality traits, extraversion, and from the dimensions of Alexithymia, difficulty in describing feelings and focusing on external experiences had a significant relationship with emotional suppression.In the whole sample, the variables of Alexithymia, total score, and difficulty dimensions in describing and identifying emotions with emotional suppression are positively and significantly correlated. This means that increasing the level of Alexithymia and the difficulty in describing and recognizing emotions increases emotional suppression scores. No significant relationship was found between the focus on exterior experiences and emotional suppression.

\section{REFERENCES}

[1] Conrad, I. Bodeewes, G. Schilling, F. Geiser, K. Imbierowicz, and R. Liedtke."Central serous chorioretinopathy and stress" Ophthalmologe, 97, 8, 527-531,2010.

[2] Jimenez SS, Niles BL \& Park CL."A mindfulness model of affect regulation and depressive symptoms: Positive emotions, mood regulation expectancies, and self-acceptance as regulatory mechanisms", Personality and Individual Differences, 49: 645-650,2010.

[3] Spahn C, Wiek J, Burger T, Hansen L."Psychosomatic aspects in patients with central serous chorioretinopathy", Br J Ophthalmol. Jun; 87(6):704-8, 2003.

[4] Aghaei, Sh. Saki, N.,Daneshmand, E.,Kardeh, B., "Prevalence of Psychological Disorders in Patients with Alopecia Areata in Comparison with Normal Subjects," ISRN Dermatology, vol., 4 pages, 2014.

[5] Roger D, Najarian B.'The construction and validation of a new scale for measuring emotion control", Personal and Individual Differences, (10): 845853,1989.

[6] Talbot, F., Nouwen, A.,"A review of the relationship between depression and diabetes in adults: is there a link?", Diabetes care 23.10 , 1556-1562, 2000.

[7] Liebert, R. M. and Spiegler, M. D. Personality: Strategies and issues, 6th, Pacific Grove, CA: Brooks/Cole, 1990.

[8] Cheng, H.,Furnham, A. "Personality, Self-Esteem, and Demographic Predictions of Happiness and Depression", Personality and Individual Differences, 34, 921-942, 2003.

[9] Fortune, D. G., Richards, H. L., Griffiths, C. E. M., \& Main, C. J., ”Targeting cognitive-behaviour therapy to patients' implicit model of psoriasis: Results from a patient preference controlled trial",British Journal of Clinical Psychology, 43, 65-82,2004.

[10] Avci, D., Kelleci,M.,"Alexithymia in patients with type 2 diabetes mellitus: the role of anxiety, depression, and glycemic control",Patient Prefer Adherence. 10: 1271-1277, Jul. 20, 2016.

[11] King LA, Emmons RA.”Conflict over emotional expression: Psychological and physical correlates", J. Personal and Soc. Psychol. (58): 864-877,1990. 
[12] Smithson M., Oden G.C.,'FFuzzy Set Theory and Applications in Psychology",In: Zimmermann HJ. (eds) Practical Applications of Fuzzy Technologies.The Handbooks of Fuzzy Sets Series, vol 6. Springer, Boston, MA, 1999.

[13] Goldberg, L. R., "An alternative description of personality The Big-Five factor structure", Journal of Personality and Social Psychology, 59(6), 1216-1229, 1990.

[14] Bagbay, R. M., Parker, J. D. A., Taylor, G. J.,"The twenty-item Alexithymia Scale: I. Item selection and cross validation of the factor structure", Journal of Psychosomatic Research, 38, 23-32, 2011.

[15] Zadeh LA."Knowledge representation in fuzzy logic", In: Yager RR, Zadeh LA, editors. An introduction to fuzzy logic applications in intelligent systems, Norwell, MA: Kluwer;1992.

[16] M. JavadFattahi H. A., Derakhshan-Barjoei P., "Heuristic Model of Cellular Learning Automata for Fuzzy Rule Extraction”, Research Journal of Applied Sciences, Engineering and Technology,Vol.4, 2012.

[17] J. Li and H. W. Lewis, "Fuzzy Clustering Algorithms - Review of the Applications," 2016 IEEE International Conference on Smart Cloud (SmartCloud), New York, NY, 2016, pp. 282-288.

[18] Frederick, HR., Waterman, D.,Lenat, D.,” Building Expert Systems”, Addison-Wesley,1983.

[19] Harbi Z. Hicks Y. Setchi R.”Clock Drawing Test Interpretation System”,Procedia Computer Sci. Dec, 2017.

Citation: E. Foroozandeh et al., "Fuzzy Logic Evaluation of Personality Profile and Alexithymia with Emotional Suppression in Patients with Central Serous Retinopathy", International Journal of Research Studies in Biosciences (IJRSB), vol. 6, no. 1, pp. 1-5, 2018. http://dx.doi.org/10.20431/2349-0365.0601001

Copyright: (C) 2018 Authors. This is an open-access article distributed under the terms of the Creative Commons Attribution License, which permits unrestricted use, distribution, and reproduction in any medium, provided the original author and source are credited. 